\title{
Lifetime of hot electrons in metals retrived from low loss EELS
}

C cile H bert*, A. Satz*, G. Kothleitner**, C. Eisenmenger-Sittner* and P. Schattschneider*

* Institute for Solid State Physics, Vienna University of Technology, Vienna, Austria

** Institute for Electron Microscopy, Graz University of Technology, Graz, Austria

\section{Introduction}

Electron dynamics in metals are well known to play a key role in a variety of physical and chemical phenomena. The investigation of low energy excited electrons helps in the understanding of many body effects in solids. In the case of hot electrons with energies larger than $1 \mathrm{eV}$ above the Fermi level electron-electron interactions provide the main inelastic scattering mechanism. These interactions yield inelastic lifetimes that are on the femtosecond time scale. With the use of $\vec{q}$ dependent EELS spectra one can get access to the loss function $\Im\left\{\frac{-1}{\epsilon(\vec{q}, E)}\right\}$ and therefore it is possible to estimate the lifetime of hot electrons.

\section{Results}

The reciprocal lifetime of the excited electron is energy dependent and directly related to the energy and momentum-transfer dependent loss function[1]

$$
\frac{1}{\tau\left(E_{p}\right)} \propto \int_{0}^{2 p} d q \int_{\theta_{\min }(q)}^{\theta_{\max }(q)} d \theta \sin (\theta) \cdot \Im\left\{\frac{-1}{\epsilon(q, E)}\right\}
$$

The q dependent loss function is the key parameter for the calculation and can be retrieved from the experiment since the differential cross section is related to the loss function by:

$$
\frac{\partial^{3} \sigma}{\partial^{2} \Omega \partial E} \propto \frac{1}{\left\{\theta^{2}+\theta_{e}^{2}\right\}} \cdot \Im\left\{\frac{-1}{\epsilon(q, E)}\right\}
$$

We used polycrystalline metallic Au samples to reccord spectra in diffraction mode at different scattering angles between the central spot and the $\{111\}$ diffraction ring.

- Multiple quasi elastic scattering was reduced with the method of Batson and Silcox [2].

- The loss function was obtained from the spectra by dividing by the angular Lorentzian distribution.

- Since we investigate metals, we use the following sum-rule for the normalization of the loss function:

$$
\int_{0}^{\infty} \Im\left\{\frac{-1}{\epsilon(q, E)}\right\} \frac{d E}{E}=\frac{\pi}{2} .
$$

Two spectral series were measured: a) Tecnai F20 FEG operated at $200 \mathrm{kV}$ : FWHM of the Zero Loss Peak without sample, $0.8 \mathrm{eV}$; FWHM of the ZLP at the largest scattering angle $1.2 \mathrm{eV}$. b) Monochromated Tecnai F20 in accelerated gun lens mode (Graz) FWHM of the ZLP without sample, $0.2 \mathrm{eV}$; FWHM of the ZLP at the largest scattering angle $0.4 \mathrm{eV}$.

For the low resolution $(\mathrm{FWHM}=1.2 \mathrm{eV})$ measurement we extrapolated the measured intensities down to $1 \mathrm{eV}$ with the help of a Wien2k simulation. In the case of the monochromatic measurements (FWHM $=0.4 \mathrm{eV}$ ) we need no tting in the low energy range.

$\mathrm{Ou}$ results are in good agreement with reported $a b$ initio calculations [3]. In the estimation of the lifetime we have used free particle states for the initial and nal hot electron states. As it is shown in [4] this choice leads to an underestimation of the lifetime for energies $\mathrm{E}<2.5 \mathrm{eV}$. 


\section{Conclusion}

- The lifetime of hot electrons in solids can be approximated by $\vec{q}$ dependent low loss spectra.

- The tting of the Wien $2 \mathrm{k}$ simulated loss function to the experimental data in the energy region $E<2.5 \mathrm{eV}$ does not lead to true values for the loss function. The lifetime is very sensitive to this error.

- For the determination of lifetimes of hot electrons for energies down to $1 \mathrm{eV}$ a monochromator system is necessary to get the appropriate energy resolution.

- EELS seems to be the best experimental access to lifetimes of hot electrons in Au for excitation energies $\mathrm{E} \geq 2.5 \mathrm{eV}$ and is therefore complementary to two photons methods.

\section{References}

[1] D. Pines, Elementary Excitations in Solids, W.A. Benjamin, Inc., New York, 1977.

[2] P. Batson and J. Silcox, Phys. Rev. B 27 (1983) p.5224.

[3] I. Campillo, J. M. Pitarke, A. Rubio, and P. M. Echenique: Phys Rev B (2000), 62, 1500 - 1503

[4] P.M.Echenique, J.M.Pitarke, E.V.Chulkov, and A.Rubio: Chemical Physics (2000), 251 1-35

\section{Figures}

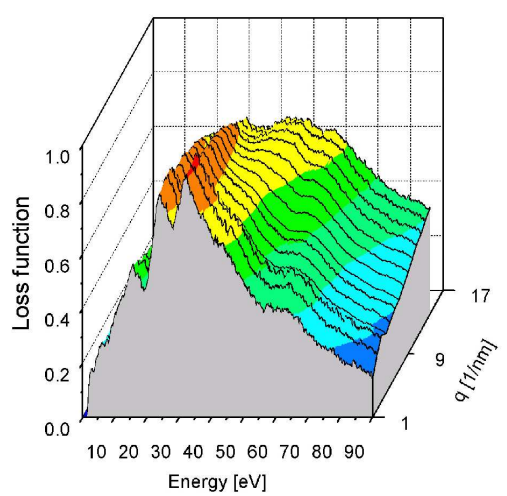

Fig. 1: Experimental loss function in $\mathrm{Au}$ as a function of wave number and energy

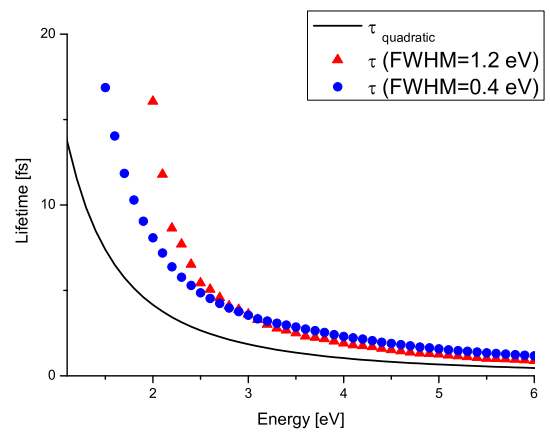

Fig. 3: Lifetime of hot electrons in Au from the present EELS experiment, compared to the quadratic approximation.

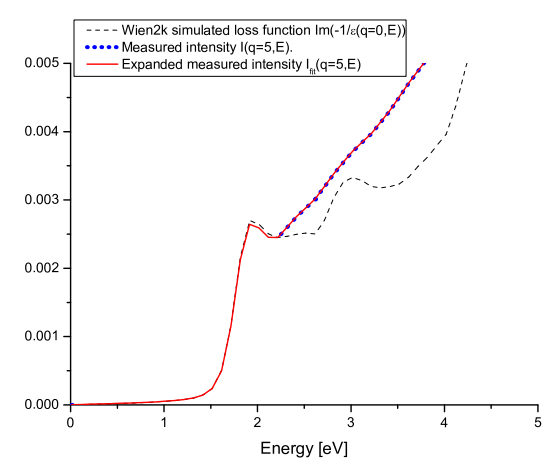

Fig. 2: Experimental spectrum for $\mathrm{q}=5 / \mathrm{nm}$ and extrapolation with the Wien $2 \mathrm{k}$ calculation.

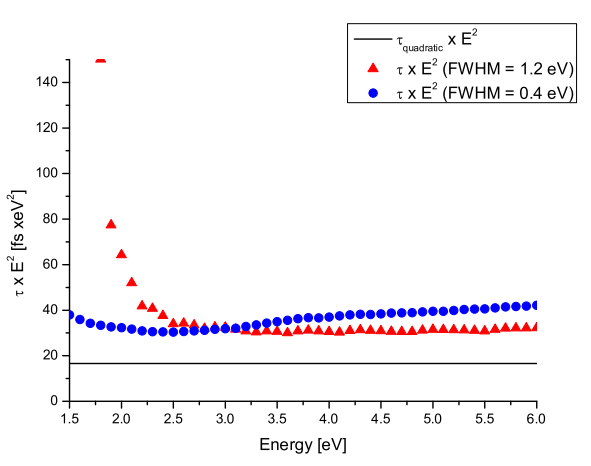

Fig. 4: Deviation from the quadratic behaviour of the lifetime of hot electrons in Au. 